\title{
SULFAMIC ACID CATALYZED FIVE COMPONENT REACTION FOR EFFICIENT AND ONE-POT SYNTHESIS OF DENSELY FUNCTIONALIZED TETRAHYDROPYRIDINE SCAFFOLD
}

\author{
ANIKET P. SARKATE, ${ }^{a} J A I P R A K A S H ~ N . ~ S A N G S H E T T I,{ }^{b}$ NANASAHEB B. DHARBALE, ${ }^{a}$ AJINKYA P. SARKATE, ${ }^{c}$ \\ DEVANAND B. SHINDE ${ }^{a^{*}}$
}

\author{
${ }^{a}$ Department of Chemical Technology, Dr. Babasaheb Ambedkar Marathwada University, Aurangabad (MS), India. \\ ${ }^{b}$ Department of Pharmaceutical Chemistry, Y B Chavan College of Pharmacy, Aurangabad (MS), India \\ ${ }^{c}$ Department of Pharmaceutical Chemistry, Poona College of Pharmacy, Bharati Vidyapeeth, Pune (MS), India
}

\begin{abstract}
The utility of sulfamic acid in One-pot assembling of aromatic aldehydes, aromatic amines and $\beta$-keto esters to afford densely functionalized tetrahydropyridines in $70-89 \%$ yield has been presented. The advantage of sulfamic acid against other catalysts in terms of yield has been studied. The effect of different solvents on reaction time and yield has also been studied. Easy work up and mild reaction condition are the advantages of the proposed method.
\end{abstract}

Keywords: Sulfamic acid, functionalized tetrahydropyridines, one-pot, five component reaction.

\section{INTRODUCTION}

Nowadays, multicomponent reactions (MCRs) ${ }^{1}$ have been rewarded a great deal attention by synthetic organic chemists throughout the world because the construction of structurally design composite molecules with an assorted range of complexity which is possible to obtain with easily available starting materials. In many cases, a single product was obtained from three or more dissimilar substrates by reacting through MCRs. ${ }^{2} \mathrm{MCR}$ possesses some advantages over conventional syntheses together with lower costs, shorter reaction time, high degrees of atom economy, the possibility for combinatorial surveying of structural variations, and environmental easiness. In the midst of prominent efforts to develop new MCRs, ${ }^{3}$ Bonfield et al ${ }^{4}$ recently reported a resourceful method for the preparation of the isoindoline framework via a six component, tandem double A3-coupling and [2+2+2] cycloaddition reaction. In addition, one-pot, multi-component reactions continue to be of attention in the synthesis of a range of composites containing particularly nitrogen atom ${ }^{5,6}$ due to the quick assembly of substituted systems lacking isolation of unstable intermediates. The significance of these one-pot reactions in such syntheses has been well established in the Mannich, Ugi, ${ }^{5}$ Biginelli, ${ }^{6}$ aza-Baylise Hillman ${ }^{7}$ reactions, and in Hantzsch dihydropyridine synthesis. ${ }^{8}$ Furthermore, the Orru group ${ }^{9}$ developed a one-pot reaction of up to eight components that involves nine new bond formations and eleven faces of multiplicity. The literature survey revealed that, MCRs that involve 1,3-dicarbonyl compounds, aldehydes, and nucleophilic compounds have received much attention because the formation of different condensation products expected depending on the specific conditions and structures of the building blocks.

The tetrahydropyridine containing gallows are broadly dispersed in natural products, biologically active molecules and organic fine chemicals. ${ }^{10} \mathrm{Compounds}$ including tetrahydropyridine motif reveal antimalarial, ${ }^{11}$ antibacterial ${ }^{12}$, antihypertensive ${ }^{13}$ anticonvulsant and anti-inflammatory activities. ${ }^{14}$ There are various synthetic strategies have been developed for the synthesis of tetrahydropyridine scaffolds, some of the them includes, imino-Diels-Alder reactions, ${ }^{15}$ aza-Prins-cyclizations, ${ }^{16}$ intramolecular Michael reactions ${ }^{17}$ and intra-molecular Mannich reaction onto iminium ions ${ }^{18}$. An alternative current strategy for the synthesis of functionalized tetrahydropyridine is using onepot MCRs. Literature survey disclose only a few methods to synthesize highly functionalized tetrahydropyridine derivatives via MCRs using catalysts including combination of L-proline/TFA ${ }^{11}$, bromodimethyl sulfonium bromide (BDMS), ${ }_{19}$ tetrabutylammonium tribromide (TBATB), ${ }^{20}$ Iodine, ${ }^{21} \mathrm{InCl}_{3,}{ }_{2}^{22}$ picric $\operatorname{acid}^{23}$ and bismuth nitrate ${ }^{24}$.

Aforesaid methods have some weaknesses like use of expensive catalyst, the amount of catalyst, failure in some of the cases to attain desired product and increased reaction time. Solid acid catalysts have proved into most significant tools for the expansion and synthesis of different chemicals. These catalysts are economic, energetic and selective.

Sulfamic acid $\left(\mathrm{H}_{2} \mathrm{NSO}_{3} \mathrm{H}\right)$ has come out as a green heterogeneous catalyst for the acid catalyzed reactions like functional group protection and deprotection, ${ }^{25}$ inter and intra-molecular imino Diels-Alder reaction, ${ }^{26}$ Pechmann condensation, ${ }^{27}$ Biginelli condensation, ${ }^{28}$ Beckmann rearrangement, ${ }^{29}$ etc. It is comparatively economic, dry, non-volatile, non hygroscopic, noncorrosive, odorless solid crystalline catalyst. It exhibits characteristic catalytic features related to its zwitter-ionic nature and hence displays an incomparable activity over a huge collection of organic conversions.

Therefore, in the present study, as a part of our ongoing research with the aforementioned catalyst, herein, we describe the use of sulfamic acid for the synthesis of functionalized tetrahydropyridine via one-pot multi-component reaction.

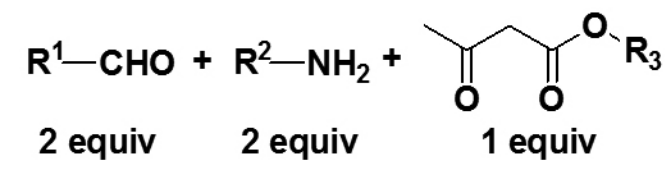

1 2

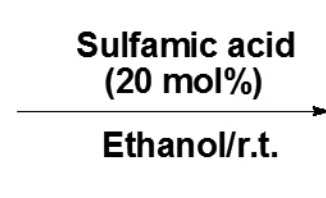

3

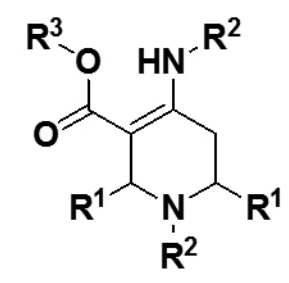

4

Scheme 1: Sulfamic acid catalyzed synthesis of densely functionalized 1,2,5,6-tetrahydropyridines. 


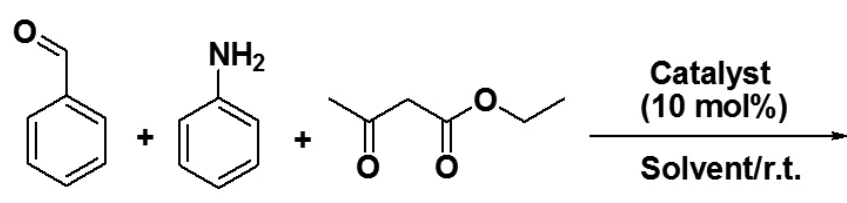

1
2

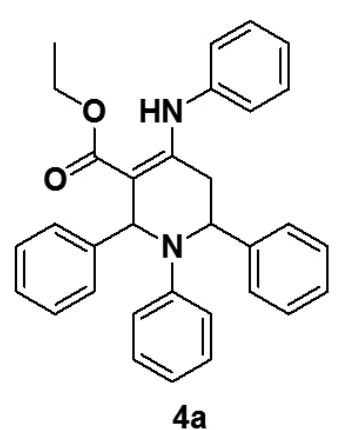

Scheme 2: Effect of different catalysts on model reaction of benzaldehyde, aniline and ethyl acetoacetate

Table 1: Effect of different catalysts on model reaction. ${ }^{\mathrm{a}}$

\begin{tabular}{|c|c|c|c|}
\hline Entry & Catalyst & Reaction time (h) & Yield $(\%)^{b}$ \\
\hline 1 & Magnessium perchlorate & 30 & 61 \\
\hline 2 & $\mathrm{HClO}_{4} / \mathrm{SiO}_{2}$ & 30 & 58 \\
\hline 3 & $\mathrm{LiClO}_{4} \cdot 3 \mathrm{H}_{2} \mathrm{O}$ & 35 & 38 \\
\hline 4 & $\mathrm{ZrOCl}_{2} .8 \mathrm{H}_{2} \mathrm{O}$ & 24 & 45 \\
\hline 5 & $\mathrm{ZrO}\left(\mathrm{NO}_{3}\right)_{2}$ & 24 & 69 \\
\hline 6 & $\mathrm{FeCl}_{3}$ & 48 & Trace \\
\hline 7 & $\mathrm{HCl}$ & 48 & Trace \\
\hline 8 & $\mathrm{CH}_{3} \mathrm{COOH}$ & 48 & Nil \\
\hline 9 & $\mathrm{CuCl}_{2}$ & 24 & 42 \\
\hline 10 & PTSA & 30 & 55 \\
\hline 11 & $\mathrm{I}_{2}$ & 16 & 65 \\
\hline 12 & Sulfamic acid & 8 & 78 \\
\hline 13 & $\mathrm{Bi}\left(\mathrm{NO}_{3}\right)_{3} \cdot 5 \mathrm{H}_{2} \mathrm{O}$ & 16 & 73 \\
\hline 14 & None & 24 & Nil \\
\hline
\end{tabular}

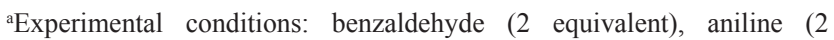
equivalent), ethylacetoacetate (1 equivalent) and sulfamic acid $(10 \mathrm{~mol} \%)$ in 5 $\mathrm{mL}$ of EtOH at room temperature. ' Isolated yield.

\section{RESULT AND DISCUSSION}

Various potential catalysts were tested for the direct synthesis of $\mathbf{4 a}$, by the model reaction of benzaldehyde ( 2 equiv), aniline ( 2 equiv) and ethyl acetoacetate (2 equiv) in ethanol at room temperature, with the results listed in Table 1. From the literature study, it has been found that lewis acid catalyst plays an important role in construction of tetrahydropyridine scaffolds. Therefore, we initiated our study with $\mathrm{Mg}\left(\mathrm{ClO}_{4}\right)_{2}$ (Table 1, Entry 1) followed by various catalysts (Table 1, Entry 2-13). Reaction of 1, 2 and $\mathbf{3}$ in the presence of catalyst under study revealed the formation of $4 \mathrm{a}$ in $38-73 \%$ yield. While, trace amounts of the product was noticed with $\mathrm{FeCl}_{3}$ and $\mathrm{HCl}$ (Table 1, Entry 6 and 7), however, no yield obtained in absence of catalysts. Amongst of catalyst tested, sulfamic acid proved to be superior for producing the better yield (78\%) of the compound 4a (Table 1, Entry 12), with reduced reaction time ( $8 \mathrm{hrs}$ ).

A review of the solvents revealed ethanol (EtOH) to be the best choice and it was used directly without rigorous drying. Whereas, considerable yields $(63 \%$ and $73 \%$ respectively) were obtained with tetrahydrofuran (THF) and acetonitrile (MeCN) (Table 2, Entry $4 \& 5$ ). Furthermore, it was also noticed that, the isolated yield of the product affected by the amount of catalyst employed for the reaction. $5 \mathrm{~mol} \%$ of the catalyst afforded yield of the isolated product in only $28 \%$ after $20 \mathrm{hrs}$ (Table 2 , Entry 1 ), while, an increase in yield was noticed up to $20 \mathrm{~mol} \%$ of the catalyst loading. The best results were obtained with $20 \mathrm{~mol} \%$ of the catalyst in ethanol in 8 hrs of reaction time (88\%, Table 2, Entry 8). No significant improvement in the results was noticed with further increase in the catalyst loading (Table 2, Entry 9). Therefore, the same tactic was employed for the further exploration of the applicability of the method by synthesizing a range of densely functionalized tetrahydropyridines (Table 3). All the synthesized compounds 4a-p, were characterized by melting point, IR, ${ }^{1} \mathrm{HNMR}$ and mass spectral analysis.

To explore the scope and generality of this five component reaction under optimized conditions, a variety of aromatic aldehydes containing electron donating groups (-Me and $-\mathrm{OMe}$ ) and electron withdrawing substituents in the aromatic ring $\left(-\mathrm{Br}-\mathrm{F},-\mathrm{Cl},-\mathrm{NO}_{2}\right.$ and $\left.-\mathrm{CN}\right)$ were reacted with varying $\beta$-keto esters and substituted amines. The reaction time and percentage yield obtained for functionalized tetrahydropyridine derivatives (4a-p) were as listed in Table 3. In common, aromatic aldehydes bearing electron-donating and electron-withdrawing functional groups at different positions reacted with ethyl acetoacetate or methyl acetoacetate efficiently in the presence of substituted anilines to generate the corresponding products in good to better yields (Table 3). However, in case of 3-nitro benzaldehyde, 2-nitro and 4-cyano benzaldehyde, the products were obtained in moderate yield (Table 3, entry $\mathbf{4 k}$, $\mathbf{4 I}$ and $\mathbf{4 m}$ respectively), possibly due to the formation of more stable imines, having an added conjugation in the presence of nitro group, which become less reactive and less soluble in ethanol.

The present protocol was also examined for substituted amine with groups like - $\mathrm{OCH}_{3}$ and 1,3-dicarbonyl compounds like ethyl and methyl acetoacetate with an assorted aldehydes and amines, wherein, the desired tetrahydropyridine derivatives were obtained in better yield as illustrated in Table 3. This established that the alkoxy $\left(-\mathrm{OR}_{3}\right)$ moiety present in the ester functionality does not have any primary role in determining the route of the reaction.

A plausible mechanism for the synthesis of functionalized tetrahydropyridine is as shown in Scheme 3.

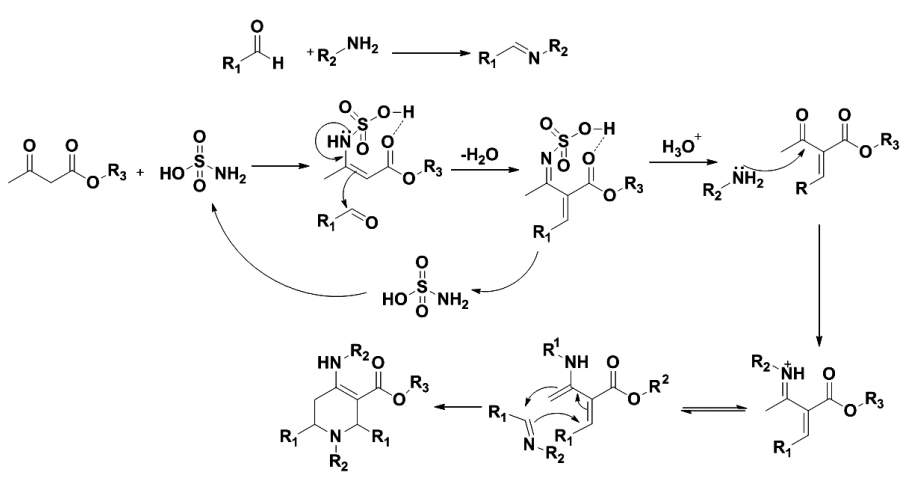

Scheme 3: Mechanism for the synthesis of tetrahydropyridines

\section{CONCLUSION}

In conclusion, we have developed a competent method for the synthesis of densely functionalized tetrahydropyridine through one pot five component reactions using a catalytic amount of sulfamic acid at room temperature. 
Table 2: Investigation of the amounts of catalyst and solvent effects on the model reaction ${ }^{\text {a }}$

\begin{tabular}{|c|c|c|c|c|}
\hline Entry & Catalyst loading (mol\%) & Solvent & Reaction Time (h) & Yield (\%) $^{\mathbf{b}}$ \\
\hline 1 & 5 & $\mathrm{H}_{2} \mathrm{O}$ & 20 & 28 \\
\hline 2 & 10 & EtOAc & 12 & 52 \\
\hline 3 & 10 & EtOAc & 12 & 48 \\
\hline 4 & 10 & THF & 14 & 63 \\
\hline 5 & 10 & MeCN & 12 & 73 \\
\hline 6 & 10 & EtOH & 8 & 83 \\
\hline 7 & 15 & EtOH & 8 & 88 \\
\hline 8 & 20 & EtOH & 8 & 84 \\
\hline 9 & 30 & EtOH & 8 & Nil \\
\hline 10 & 20 & Neat & 12 & 83 \\
\hline
\end{tabular}

${ }^{a}$ Experimental conditions: benzaldehyde ( 2 equivalent), aniline ( 2 equivalent), ethylacetoacetate ( 1 equivalent) and sulfamic acid in $5 \mathrm{~mL}$ of solvent at room temperature. ${ }^{b}$ Isolated yield.

Table 3: Sulfamic acid catalyzed synthesis of functionalized tetrahydropyridine scaffolds.

\begin{tabular}{|c|c|c|c|c|c|c|c|}
\hline \multirow{2}{*}{ Entry } & \multirow{2}{*}{$\mathbf{R}_{1}$} & \multirow{2}{*}{$\mathbf{R}_{2}$} & \multirow{2}{*}{$\mathbf{R}_{3}$} & \multirow{2}{*}{ Time (h) } & \multirow{2}{*}{ Yield (\%) } & \multicolumn{2}{|c|}{ Melting Point $\left({ }^{\circ} \mathrm{C}\right)$} \\
\hline & & & & & & Observed & Reported \\
\hline $4 a$ & $\mathrm{Ph}$ & $\mathrm{Ph}$ & Et & 08 & 88 & $175-176$ & -- \\
\hline $4 b$ & $4-\mathrm{CH}_{3} \mathrm{C}_{6} \mathrm{H}_{4}$ & $\mathrm{Ph}$ & Et & 10 & 86 & $213-215$ & -- \\
\hline $4 c$ & $4-\mathrm{OCH}_{3} \mathrm{C}_{6} \mathrm{H}_{4}$ & $\mathrm{Ph}$ & Et & 10 & 81 & $180-181$ & -- \\
\hline $4 d$ & $3,4,5-\left(\mathrm{OCH}_{3}\right)_{3} \mathrm{C}_{6} \mathrm{H}_{2}$ & $\mathrm{Ph}$ & $\mathrm{Et}$ & 09 & 79 & $167-168$ & -- \\
\hline $4 e$ & $2-\mathrm{OCH}_{3} \mathrm{C}_{6} \mathrm{H}_{4}$ & $\mathrm{Ph}$ & $\mathrm{Me}$ & 09 & 75 & $247-249$ & -- \\
\hline $4 f$ & $4-\mathrm{ClC}_{6} \mathrm{H}_{4}$ & $\mathrm{Ph}$ & $\mathrm{Et}$ & 08 & 78 & $189-190$ & -- \\
\hline $4 \mathrm{~g}$ & $4-\mathrm{BrC}_{6} \mathrm{H}_{4}$ & $4-\mathrm{OCH}_{3} \mathrm{C}_{6} \mathrm{H}_{4}$ & $\mathrm{Me}$ & 11 & 80 & $177-179$ & $179^{11}$ \\
\hline $4 h$ & $3-\mathrm{ClC}_{6} \mathrm{H}_{4}$ & 4- $\mathrm{OCH}_{3} \mathrm{C}_{6} \mathrm{H}_{4}$ & $\mathrm{Me}$ & 12 & 73 & $160-161$ & $160^{11}$ \\
\hline $4 i$ & 4- $\mathrm{FC}_{6} \mathrm{H}_{4}$ & 4- $\mathrm{OCH}_{3} \mathrm{C}_{6} \mathrm{H}_{4}$ & $\mathrm{Me}$ & 12 & 70 & 202-204 & $205^{11}$ \\
\hline $4 j$ & $4-\mathrm{NO}_{2} \mathrm{C}_{6} \mathrm{H}_{4}$ & $\mathrm{Ph}$ & $\mathrm{Me}$ & 11 & 85 & $235-236$ & -- \\
\hline $4 k$ & $3-\mathrm{NO}_{2} \mathrm{C}_{6} \mathrm{H}_{4}$ & $\mathrm{Ph}$ & $\mathrm{Me}$ & 12 & 77 & $183-184$ & $180^{11}$ \\
\hline 41 & $2-\mathrm{NO}_{2} \mathrm{C}_{6} \mathrm{H}_{4}$ & $\mathrm{Ph}$ & $\mathrm{Me}$ & 12 & 71 & $225-227$ & -- \\
\hline $4 \mathrm{~m}$ & 4- $\mathrm{CNC}_{6} \mathrm{H}_{4}$ & 4- $\mathrm{OCH}_{3} \mathrm{C}_{6} \mathrm{H}_{4}$ & $\mathrm{Et}$ & 10 & 73 & $211-213$ & -- \\
\hline $4 n$ & $\mathrm{Ph}$ & 4- $\mathrm{OCH}_{3} \mathrm{C}_{6} \mathrm{H}_{4}$ & $\mathrm{Me}$ & 09 & 80 & $220-222$ & -- \\
\hline 40 & 4- $\mathrm{CH}_{3} \mathrm{C}_{6} \mathrm{H}_{4}$ & 4- $\mathrm{OCH}_{3} \mathrm{C}_{6} \mathrm{H}_{4}$ & Et & 10 & 89 & $223-225$ & -- \\
\hline $4 p$ & $4-\mathrm{OCH}_{3} \mathrm{C}_{6} \mathrm{H}_{4}$ & 4- $\mathrm{OCH}_{3} \mathrm{C}_{6} \mathrm{H}_{4}$ & $\mathrm{Et}$ & 10 & 83 & $178-180$ & -- \\
\hline
\end{tabular}

The recognized quality of this method, encompass gentle reaction conditions, high atom efficiency, economical starting materials, uncontaminated reaction profiles and environmentally friendly catalyst.

\section{EXPERIMENTAL}

All the chemicals \& solvents were used from Sigma-Aldrich. Melting points were uncorrected $\&$ recorded on optimelt digital melting point apparatus. IR spectra were recorded on Bruker Alpha E FTIR spectrophotometer. ${ }^{1} \mathrm{H}$ NMR was recorded on Varian $300 \mathrm{MHz}$ spectrometer by using TMS as an internal standard. Molecular weight was determined using LC-MS Scinpor ESI.

General procedure for the synthesis of (4a-p)

In a $100 \mathrm{~mL}$ flask, amine $(1.0 \mathrm{mmol})$, acetoacetic ester $(0.5 \mathrm{mmol})$, sulfamic acid $(0.1 \mathrm{mmol})$ and ethanol $(5 \mathrm{~mL})$ were added sequentially, followed by aromatic aldehyde $(1.0 \mathrm{mmol})$. The reaction mixture was then stirred at room temperature until completion of the reaction ( $8-12 \mathrm{hrs}$, as monitored by TLC). A solid appeared in the reaction mixture was filtered and thoroughly washed with aqueous ethanol to afford the desired pure product 4 . The same procedure was followed for the synthesis of all other functionalized tetrahydropyridine (4a-p). Crude products were further purified by column chromatography on silica gel (EtOAc/pet. ether) to give a pure product. Spectral evaluation of compounds:

Ethyl carboxylate $(4 a)$ :

1,2,6-triphenyl-4-(phenylamino)tetrahydropyridine-3-

${ }^{1} \mathbf{H}$ NMR (300 MHz, $\mathbf{C D C l}_{3}$ ) $\delta: 1.29\left(\mathrm{t}, 3 \mathrm{H}, \mathrm{CH}_{3}\right), 4.12\left(\mathrm{q}, 2 \mathrm{H}, \mathrm{O}-\mathrm{CH}_{2}\right), 4.1$ (s, $1 \mathrm{H}, \mathrm{NH}), 2.34\left(\mathrm{~d}, 2 \mathrm{H}, \mathrm{CH}_{2}\right) 4.28(\mathrm{t}, 1 \mathrm{H}, \mathrm{CH}), 4.49(\mathrm{~s}, 1 \mathrm{H}, \mathrm{CH}), 6.43-7.22$ (m, 5H, HAr), 6.34-6.92 (m, 5H, HAr), 7.02-7.19 (m, 5H, HAr), 7.10-7.27 (m, 5H, HAr); IR (KBr, v/ $\left.\mathbf{c m}^{-1}\right): 3267,1744,1545,1155,1243$; LC-MS (m/z): $474.11\left(\mathrm{M}^{+}\right)$.

Ethyl

1,2,5,6-tetrahydro-1-phenyl-4-(phenylamino)-2,6-diptolylpyridine-3-carboxylate(4b):

${ }^{1} \mathbf{H}$ NMR: (300 MHz, $\left.\mathbf{C D C l}\right) \boldsymbol{\delta}: 1.22\left(\mathrm{t}, 3 \mathrm{H}, \mathrm{CH}_{3}\right), 4.03\left(\mathrm{q}, 2 \mathrm{H}, \mathrm{O}-\mathrm{CH}_{2}\right)$, $2.24\left(\mathrm{~d}, 2 \mathrm{H}, \mathrm{CH}_{2}\right), 2.31\left(\mathrm{~s}, 6 \mathrm{H}, \mathrm{CH}_{3}\right), 3.97(\mathrm{~s}, 1 \mathrm{H}, \mathrm{NH}), 4.25(\mathrm{t}, 1 \mathrm{H}, \mathrm{CH}), 4.55$ (s, 1H, CH), 6.53-7.08 (m, 5H, HAr), 6.41-7.01 (m, 5H, HAr), 6.94-7.10 (m, 4H, HAr), 6.99-7.03 (m, 4H, HAr); IR (KBr, v/ $\left.\mathbf{c m}^{-1}\right): 3243,1743,1490,1156$, 1252; LC-MS (m/z): $502.43\left(\mathrm{M}^{+}\right)$. 
Ethyl2,6-bis(4-methoxyphenyl)-1-phenyl-4-(phenylamino) tetrahydropyridine-3-carboxylate(4c):

${ }^{1} \mathbf{H}$ NMR (300 MHz, $\mathbf{C D C l}_{3}$ ) $\boldsymbol{\delta}: 1.24\left(\mathrm{t}, 3 \mathrm{H}, \mathrm{CH}_{3}\right), 4.08\left(\mathrm{q}, 2 \mathrm{H}, \mathrm{O}_{-} \mathrm{CH}_{2}\right)$, $2.31\left(\mathrm{~d}, 2 \mathrm{H}, \mathrm{CH}_{2}\right), 3.63\left(\mathrm{~s}, 6 \mathrm{H}, \mathrm{OCH}_{3}\right), 4.02(\mathrm{~s}, 1 \mathrm{H}, \mathrm{NH}), 4.09(\mathrm{t}, 1 \mathrm{H}, \mathrm{CH}), 4.67$ (s, 1H, CH), 6.59-6.98 (m, 5H, HAr), 6.37-6.91 (m, 5H, HAr), 6.65-7.03 (m, 4H, HAr), 6.66-7.08 (m, 4H, HAr); IR (KBr, v/ $\left.\mathbf{c m}^{-1}\right): 3251,1741,1520,1185$, 1223; LC-MS (m/z): $536.21\left(\mathrm{M}^{+}\right)$

Ethyl2,6-bis(3,4,5-trimethoxyphenyl)-1-phenyl-4-(phenylamino) tetrahydropyridine-3-carboxylate $(4 d)$ :

${ }^{1} \mathbf{H}$ NMR (300 MHz, $\mathbf{C D C l}_{3}$ ) $\delta: 1.22\left(\mathrm{t}, 3 \mathrm{H}, \mathrm{CH}_{3}\right), 4.06$ (q, 2H, O-CH $)$, $2.29\left(\mathrm{~d}, 2 \mathrm{H}, \mathrm{CH}_{2}\right), 3.7\left(\mathrm{~s}, 18 \mathrm{H}, \mathrm{OCH}_{3}\right), 4.03(\mathrm{~s}, 1 \mathrm{H}, \mathrm{NH}), 4.01(\mathrm{t}, 1 \mathrm{H}, \mathrm{CH})$, 4.7 (s, 1H, CH), 6.6-7.08 (m, 5H, HAr), 6.41-7.01 (m, 5H, HAr),6.03 (s, 2H, HAr), 6.03 (s, 2H, HAr); IR (KBr, v/cm $\left.{ }^{-1}\right): 3243,1737,1521,1156,1243$; LC-MS (m/z): $656.41\left(\mathrm{M}^{+}\right)$.

Ethyl2,6-bis(2-methoxyphenyl)-1-phenyl-4-(phenylamino) tetrahydropyridine-3-carboxylate $(4 e)$ :

${ }^{1}$ H NMR (300 MHz, $\mathbf{C D C l}_{3}$ ) $\boldsymbol{\delta}: 3.52\left(\mathrm{~s}, 3 \mathrm{H}, \mathrm{CH}_{3}\right), 2.33\left(\mathrm{~d}, 2 \mathrm{H}, \mathrm{CH}_{2}\right), 3.68$ $\left(\mathrm{s}, 6 \mathrm{H}, \mathrm{OCH}_{3}\right), 4.01(\mathrm{~s}, 1 \mathrm{H}, \mathrm{NH}), 3.87(\mathrm{t}, 1 \mathrm{H}, \mathrm{CH}), 4.67(\mathrm{~s}, 1 \mathrm{H}, \mathrm{CH}), 6.51-7.11$ (m, 5H, HAr), 6.43-7.04 (m, 5H, HAr), 6.73- 6.97 (s, 4H, HAr), 6.67- 6.97 (s, 4H, HAr); IR (KBr, v/cm $\left.{ }^{-1}\right): 3307,1743,1540,1152,1210$; LC-MS (m/z): $522.11\left(\mathrm{M}^{+}\right)$.

Ethyl 2,6-bis(4-chlorophenyl)-1-phenyl-4-(phenylamino) tetrahydropyridine-3-carboxylate $(4 f)$ :

${ }^{1} \mathbf{H}$ NMR (300 MHz, CDCl) $\boldsymbol{\delta}: 1.32\left(\mathrm{t}, 3 \mathrm{H}, \mathrm{CH}_{3}\right), 4.02\left(\mathrm{q}, 2 \mathrm{H},-\mathrm{O}-\mathrm{CH}_{2}\right)$, $2.37\left(\mathrm{~d}, 2 \mathrm{H}, \mathrm{CH}_{2}\right), 4.09(\mathrm{~s}, 1 \mathrm{H}, \mathrm{NH}), 4.0(\mathrm{t}, 1 \mathrm{H}, \mathrm{CH}), 4.67(\mathrm{~s}, 1 \mathrm{H}, \mathrm{CH}), 6.51-$ 7.11 (m, 5H, HAr), 6.43-7.04 (m, 5H, HAr), 6.73-6.97 (s, 4H, HAr), 6.67-6.97 (s, 4H, HAr); IR (KBr, v/cm-1): 3307, 1743, 1540, 1152, 1210; LC-MS (m/z): $544.19\left(\mathrm{M}^{+}\right)$.

Methyl4-(4-methoxyphenylamino)-2,6-bis(4-bromophenyl)-1-(4methoxyphenyl) tetrahydro pyridine-3-carboxylate(4g):

${ }^{1} \mathrm{H}$ NMR (300 MHz, CDCl $)$ d: $3.58\left(\mathrm{~s}, 3 \mathrm{H}, \mathrm{O}_{-} \mathrm{CH}_{3}\right), 3.67\left(\mathrm{~s}, 6 \mathrm{H}, \mathrm{OCH}_{3}\right)$, $4.1\left(\mathrm{q}, 2 \mathrm{H},-\mathrm{O}-\mathrm{CH}_{2}\right), 2.36\left(\mathrm{~d}, 2 \mathrm{H}, \mathrm{CH}_{2}\right), 4.62(\mathrm{~s}, 1 \mathrm{H}, \mathrm{CH}), 3.89(\mathrm{~s}, 1 \mathrm{H}, \mathrm{NH})$, 6.43-6.65 (m, 4H, HAr), 6.33-6.55 (m, 4H, HAr),6.89-7.45 (m, 4H, HAr), 7.10-7.46 (m, 4H, HAr); IR (KBr, v/ $\mathbf{c m}^{-1}$ ): 3307, 1743, 1545, 1155, 1232; LC-MS (m/z): $678.18\left(\mathrm{M}^{+}\right)$.

Methyl-4-(4-methoxyphenylamino)-2,6-bis(3-chlorophenyl)-1,2,5,6tetrahydro-1-(4-methoxyphenyl)pyridine-3-carboxylate(4h):

${ }^{1} \mathrm{H}$ NMR (300 MHz, CDCl$)$ d: $3.72\left(\mathrm{~s}, 3 \mathrm{H},-\mathrm{O}-\mathrm{CH}_{3}\right), 2.27\left(\mathrm{~d}, 2 \mathrm{H}, \mathrm{CH}_{2}\right)$, $3.69\left(\mathrm{~s}, 6 \mathrm{H}, \mathrm{OCH}_{3}\right), 4.14\left(\mathrm{t}, 2 \mathrm{H}, \mathrm{CH}_{2}\right), 4.56(\mathrm{~s}, 1 \mathrm{H}, \mathrm{CH}), 3.92(\mathrm{~s}, 1 \mathrm{H}, \mathrm{NH})$, 6.44-6.63 (m, 4H, HAr), 6.30-6.48 (m, 4H, HAr),6.97-7.04 (m, 4H, HAr), 7.03-7.19 (m, 4H, HAr); IR (KBr, v/cm ${ }^{-1}$ ): 3276, 1736, 1547, 1167, 1256; LC-MS (m/z): $588.11\left(\mathrm{M}^{+}\right)$.

Methyl-4-(4-methoxyphenylamino)-2,6-bis(4-fluorophenyl)-1,2,5,6tetrahydro-1-(4-methoxyphenyl)pyridine-3-carboxylate(4i):

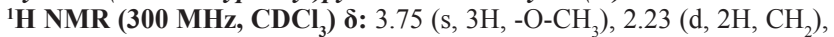
$3.70\left(\mathrm{~s}, 6 \mathrm{H}, \mathrm{OCH}_{3}\right), 4.18\left(\mathrm{t}, 2 \mathrm{H}, \mathrm{CH}_{2}\right), 4.51(\mathrm{~s}, 1 \mathrm{H}, \mathrm{CH}), 3.98(\mathrm{~s}, 1 \mathrm{H}, \mathrm{NH})$, 6.45-6.60 (m, 4H, HAr), 6.32-6.47 (m, 4H, HAr),6.89-7.01 (m, 4 H, HAr), 6.97-7.09 (m, 4H, HAr); IR (KBr, v/cm $\left.{ }^{-1}\right): 3280,1738,1537,1170,1267$; LC-MS (m/z): $556.18\left(\mathrm{M}^{+}\right)$.

Methyl-1,2,5,6-tetrahydro-2,6-bis(4-nitrophenyl)-1-phenyl-4(phenylamino)pyridine-3-carboxylate( $4 j)$ :

${ }^{1} \mathbf{H}$ NMR (300 MHz, CDCl$)$ ): $3.74\left(\mathrm{~s}, 3 \mathrm{H},-\mathrm{O}-\mathrm{CH}_{3}\right), 2.39\left(\mathrm{~d}, 2 \mathrm{H}, \mathrm{CH}_{2}\right)$, $4.09\left(\mathrm{t}, 1 \mathrm{H}, \mathrm{CH}_{2}\right), 4.57(\mathrm{~s}, 1 \mathrm{H}, \mathrm{CH}), 4.01(\mathrm{~s}, 1 \mathrm{H}, \mathrm{NH}), 6.58-7.02(\mathrm{~m}, 5 \mathrm{H}, \mathrm{HAr})$, 6.41-6.95 (m, 5H, HAr), 7.29-8.01 (m, 4H, HAr), 7.33-8.09 (m, 4H, HAr); IR (KBr, v/ $\left.\mathbf{c m}^{-1}\right): 3267,1741,1556,1164,1275,1352,1580$; LC-MS (m/z): $550.12\left(\mathrm{M}^{+}\right)$

Methyl-1,2,5,6-tetrahydro-2,6-bis(3-nitrophenyl)-1-phenyl-4(phenylamino)pyridine-3-carboxylate $(4 k)$ :

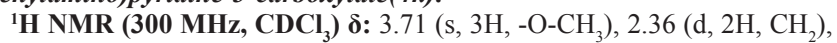
$4.09\left(\mathrm{t}, 1 \mathrm{H}, \mathrm{CH}_{2}\right), 4.61(\mathrm{~s}, 1 \mathrm{H}, \mathrm{CH}), 4.05(\mathrm{~s}, 1 \mathrm{H}, \mathrm{NH}), 6.57-6.96(\mathrm{~m}, 5 \mathrm{H}, \mathrm{HAr})$, 6.49-7.07 (m, 5H, HAr), 7.38-8.00 (m, 4H, HAr), 7.39-8.02 (m, 4H, HAr); IR (KBr, v/cm $\left.{ }^{-1}\right): 3284,1734,1555,1172,1281,1350,1520 ; \mathbf{L C}-\mathbf{M S}(\mathbf{m} / \mathbf{z}):$ $550.12\left(\mathrm{M}^{+}\right)$.

methyl-1,2,5,6-tetrahydro-2,6-bis(3-nitrophenyl)-1-phenyl-4(phenylamino)pyridine-3-carboxylate (4l):

${ }^{1} \mathbf{H}$ NMR (300 MHz, $\mathbf{C D C l}_{3}$ ) $\delta: 3.69\left(\mathrm{~s}, 3 \mathrm{H},-\mathrm{O}-\mathrm{CH}_{3}\right), 2.36\left(\mathrm{~d}, 2 \mathrm{H}, \mathrm{CH}_{2}\right)$, 4.19 (t, 1H, CH), $4.6(\mathrm{~s}, 1 \mathrm{H}, \mathrm{CH}), 4.00(\mathrm{~s}, 1 \mathrm{H}, \mathrm{NH}), 6.56-7.02$ (m, 5H, HAr), 6.47-7.02 (m, 5H, HAr), 7.40-7.98 (m, 4H, HAr), 7.43-8.04 (m, 4H, HAr); IR (KBr, v/cm $\left.{ }^{-1}\right): 3274,1738,1554,1163,1267,1351,1590 ; \mathbf{L C}-\mathbf{M S}(\mathbf{m} / \mathbf{z}):$ $550.19\left(\mathrm{M}^{+}\right)$.

ethyl-4-(4-methoxyphenylamino)-2,6-bis(4-cyanophenyl)-1,2,5,6tetrahydro-1-(4-methoxyphenyl)pyridine-3-carboxylate $(4 \mathrm{~m})$ :

${ }^{1} \mathbf{H}$ NMR (300 MHz, $\mathbf{C D C l}_{3}$ ) $\delta: 1.27\left(\mathrm{t}, 3 \mathrm{H}, \mathrm{CH}_{3}\right), 4.09$ (q, 2H, $\left.-\mathrm{O}_{-} \mathrm{CH}_{2}\right)$, $2.34\left(\mathrm{~d}, 2 \mathrm{H}, \mathrm{CH}_{2}\right), 3.69\left(\mathrm{~s}, 6 \mathrm{H},-\mathrm{OCH}_{3}\right), 4.14(\mathrm{t}, 1 \mathrm{H}, \mathrm{CH}), 4.54(\mathrm{~s}, 1 \mathrm{H}, \mathrm{CH})$ 4.05(s, 1H, NH), 6.49-6.67 (m, 4H, HAr), 6.34-6.49 (m, 4H, HAr), 7.20-7.38 (m, 4H, HAr), 7.34-7.40 (m, 4H, HAr); IR (KBr, v/cm $\left.\mathbf{c m}^{-1}\right): 3291,1742,2245$, 1153, 1263, 1576; LC-MS (m/z): $584.21\left(\mathrm{M}^{+}\right)$.

methyl-4-(4-methoxyphenylamino)-1,2,5,6-tetrahydro-1-(4methoxyphenyl)-2,6-diphenylpyridine-3-carboxylate $(4 n)$ :

${ }^{1} \mathrm{H}$ NMR (300 MHz, $\mathbf{C D C l}_{3}$ ) $8: 3.79$ (s, 3H, $\left.-\mathrm{O}-\mathrm{CH}_{3}\right), 2.26\left(\mathrm{~d}, 2 \mathrm{H}, \mathrm{CH}_{2}\right)$, $3.71\left(\mathrm{~s}, 6 \mathrm{H},-\mathrm{OCH}_{3}\right), 4.18(\mathrm{t}, 1 \mathrm{H}, \mathrm{CH}), 4.61(\mathrm{~s}, 1 \mathrm{H}, \mathrm{CH}), 4.0(\mathrm{~s}, 1 \mathrm{H}, \mathrm{NH})$, 6.44-6.64 (m, 4H, HAr), 6.37-6.53 (m, 4H, HAr), 7.03-7.11 (m, 5H, HAr), 7.09-7.16 (m, 5H, HAr); IR (KBr, v/cm $\left.{ }^{-1}\right): 3259,1732,1143,1276,1519$; LC-MS (m/z): $520.16\left(\mathrm{M}^{+}\right)$.

Ethyl-4-(4-methoxyphenylamino)-1,2,5,6-tetrahydro-1-(4methoxyphenyl)-2,6-dip-tolylpyridine-3 carboxylate(4o):

${ }^{1} \mathbf{H}$ NMR (300 MHz, CDCl $\left.)_{3}\right)$ d: $1.26\left(\mathrm{t}, 3 \mathrm{H}, \mathrm{CH}_{3}\right), 4.11\left(\mathrm{q}, 2 \mathrm{H},-\mathrm{O}-\mathrm{CH}_{2}\right)$, $2.28\left(\mathrm{~d}, 2 \mathrm{H}, \mathrm{CH}_{2}\right), 2.31\left(\mathrm{~s}, 6 \mathrm{H}, \mathrm{CH}_{3}\right), 3.73\left(\mathrm{~s}, 6 \mathrm{H},-\mathrm{OCH}_{3}\right), 4.13(\mathrm{t}, 1 \mathrm{H}, \mathrm{CH})$, $4.57(\mathrm{~s}, 1 \mathrm{H}, \mathrm{CH}), 3.96(\mathrm{~s}, 1 \mathrm{H}, \mathrm{NH}), 6.46-6.60(\mathrm{~m}, 4 \mathrm{H}, \mathrm{HAr}), 6.91(\mathrm{~m}, 4 \mathrm{H}$, HAr), 7.03 (m, 4H, HAr), 7.04 (m, 4H, HAr); IR (KBr, v/cm $\left.{ }^{-1}\right): 3263,1734$, 1145, 1266, 1519; LC-MS (m/z): $562.23\left(\mathrm{M}^{+}\right)$.

Ethyl-4-(4-methoxyphenylamino)-1,2,5,6-tetrahydro-1,2,6-tris(4methoxyphenyl)pyridine-3-carboxylate( $4 p)$ :

${ }^{1} \mathrm{H}$ NMR (300 MHz, $\left.\mathbf{C D C l}_{3}\right)$ \&: $1.28\left(\mathrm{t}, 3 \mathrm{H}, \mathrm{CH}_{3}\right), 4.17\left(\mathrm{q}, 2 \mathrm{H},-\mathrm{O}_{-} \mathrm{CH}_{2}\right)$, $2.28\left(\mathrm{~d}, 2 \mathrm{H}, \mathrm{CH}_{2}\right), 3.68\left(\mathrm{~s}, 12 \mathrm{H},-\mathrm{OCH}_{3}\right), 4.19(\mathrm{t}, 1 \mathrm{H}, \mathrm{CH}), 4.57(\mathrm{~s}, 1 \mathrm{H}, \mathrm{CH})$ 4.01(s, 1H, NH), 6.46-6.57 (m, 4H, HAr), 6.38-6.54 (m, 4H, HAr), 6.61-6.92 (m, 4H, HAr), 6.75-6.99 (m, 4H, HAr); IR (KBr, v/ $\left.\mathbf{c m}^{-1}\right)$ : 3265, 1735, 1145 , 1271, 1524; LC-MS (m/z): $594.16\left(\mathrm{M}^{+}\right)$

\section{ACKNOWLEDGEMENT}

The authors are thankful to the Head, Department of Chemical Technology, Dr. Babasaheb Ambedkar Marathwada University, Aurangabad 431004 (MS), India, for providing the laboratory facility.

\section{REFERENCES}

1. D. J. Ramón, and M. Yus, Angew. Chem. Int. Ed., 44, 1602 (2005).

2. H. Fujioka, K. Murai, O. Kubo, Y. Ohba, and Y. Kita, Org. Lett., 9, 1687 (2007).

3. K. Kumaravel, and G.Vasuki, Curr.Org. Chem., 13, 1820 (2009).

4. E. R. Bonfield, and C. J. Li, Adv. Synth. Catal., 350, 370 (2008).

5. I. Ugi, A. Domling, and B. J. Werner, Heterocycl. Chem., 37, 647 (2000).

6. C. O. Kappe, Eur. J. Med. Chem., 35, 1043 (2000).

7. D. Balan, and H. Adolfsson, Tet.Lett., 44, 2521 (2003).

8. G.Jones, Comprehensive Heterocyclic Chemistry, Pergamon, Oxford, Vol. 2, (1984), p 482.

9. N. Elders, D. vanderBorn, L. J. D. Hendrickx, B. J .J. Timmer, A. Krause E. Janssen, F. J. J. de Kanter, E. Ruijter, and R. V. A. Orru, Angew. Chem. Int. Ed., 48, 5856 (2009).

10. J. W. Daly, T. F. Spande, and H. M.Garraffo, J. Nat. Prod., 68, 1556 (2005).

11. M. Misra, S. K. Pandey, V. P. Pandey, J. Pandey, R. Tripathi, and R. P. Tripathi, Bioorg. Med. Chem., 17, 625 (2009).

12. Y. Zhou, V. E. Gregor, B. K. Ayida, G. C. Winters, Z. Sun, D. Murphy, G.Haley, D. Bailey, J. M. Froelich, S. Fish, S. E. Webber, T. Hermann, and D. Wall, Bioorg. Med. Chem. Lett., 17, 1206 (2007).

13. S. Petit, J. P. Nallet, M. Guillard, J. Dreux, R. Chermat, M. Poncelet, C. Bulach, P. Simon, C. Fontaine, M. Barthelmebs, and J. L. Imbs, Eur.J.Med. Chem., 26, 19 (1991).

14. H. Bin, A. M. Crider, and J. P. Stables, Eur. J. Med. Chem., 36, 265 (2001).

15. M. Sales, and A. B. Charette, Org. Lett., 7, 5773 (2005).

16. R. M. Carballo, M. A. Ramirez, M. L. Rodriguez, V. S. Martin, and J. I. Padron, Org. Lett., 8, 3837 (2006).

17. S. Fustero, D. Jimenez, J. Moscardo, S. Catalan, and C. D. Pozo, Org. Lett., 9, 5283 (2007).

18. F. A. Davis, B. Chao, and A. Rao, Org. Lett., 3, 3169 (2001).

19. A. T. Khan, T. Parvin, and L. H. Choudhury, J. Org. Chem., 73, 8393 (2008).

20. A. T. Khan, M. Lal, and M. M. Khan, Tet.Lett., 51, 4419 (2010).

21. A. T. Khan, M. M. Khan, and K. K. R. Bannuru, Tet. Lett., 66, 7762 (2010).

22. P. A. Clarke, A. V. Zaytzev, and A. C. Whitwood, Tet. Lett., 48, 5209 (2007).

23. C. Mukhopadhyay, S. R. Ray, J. Butcher, and A. M. Schmiedekamp, Tet. Lett., 52, 5835 (2011). 
24. G. Brahmachari, and S. Das, Tet. Lett., 53, 1479 (2012).

25. T. S. Jin, G. Sun, Y. W. Li, and T. S. Li, Green Chem., 4, 255 (2002).

26. R. Nagarajan, C. J. Magesh, and P. T. Perumal, Synthesis, 1, 69 (2004).

27. P. R. Sing, D. U. Singh, and S. D. Samant, Synlett, 11, 1909 (2004).
28. J. T. Li, J. F. Han, J. H. Yang, and T. S. Li, Ultrason. Sonochem., 10, 119 (2003).

29. B. Wang, Y. L. Gu, G. Y. Luo, T. Yang, L. M. Yang, and J. S. Suo, Tet. Lett., 45, 3369 (2004). 